\title{
Dependency, the Thermoelectric Figure of Merit of a Material Consisting of Particles on the Parameters of a Material
}

\author{
F. V. Kharlamov, V. F. Kharlamov \\ State University, Education-Science-Production Complex, Orel, Russia \\ Email: vladkharl@rambler.ru
}

Received 12 August 2014; revised 10 September 2014; accepted 17 September 2014

Copyright (C) 2014 by authors and Scientific Research Publishing Inc.

This work is licensed under the Creative Commons Attribution International License (CC BY). http://creativecommons.org/licenses/by/4.0/

c) (i) Dpen Access

\begin{abstract}
The properties of a ball-shaped semiconductor particles and metal particles with a semiconductor thin film on the surface thereof are established. So the dimensionless thermoelectric figure of merit of a material consisting of a large number of these particles is equal to $10-100$.
\end{abstract}

Keywords

Thermoelectric Effect, Energy Transformation, Semiconductor, Particles

\section{Introduction}

The efficiency of the direct conversion of heat energy into electric current energy using solids is characterized by the dimensionless thermoelectric figure of merit of a material $Z=\sigma \alpha^{2} T / k$, where $\sigma, \alpha, T$ and $k$ are the electrical conductivity, differential thermopower, absolute temperature and thermal conductivity of the material, respectively. Numerous and long-term studies have not resulted yet in developing materials with $Z>2$ (see, for instance, [1]-[9]).

In this paper we discuss the problem of a material with $Z \gg 1$.

\section{Model and Its Research}

We shall consider the thermoelectric properties of a material consisting of a large number of identical ballshaped non-degenerate $n$-type semiconductor particles contacting each other and metal particles with a $n$-type semiconductor thin film on the surface thereof. The phonon component of the material's thermal conductivity is zero as the point contact area is zero. This type of material has electronic thermal conductivity made possible by 
electron tunneling through the vacuum gaps between the particles near the point contacts. We shall calculate the $Z$ parameter of this material. We shall simplify the task and assume that the particles make a simple cubical lattice, also we shall assume the material temperature $T=300-800 \mathrm{~K}$ and the particles diameter $d=10^{-6}-10^{-3} \mathrm{~m}$.

We shall offer the following symbols:

$$
D[\varepsilon, l(r)] \approx \exp \left\{-2 l(r)\left[2 m_{0}(\chi-\varepsilon)\right]^{1 / 2} / \hbar\right\}
$$

is the potential barrier transparency coefficient for the electrons tunneling from particle into particle through the gap between them; $\varepsilon$ is the energy of electrons in a solid; $l(r)=2 r^{2} / d$ is the vacuum gap between the particles at the contact between them; $r$ being the distance from the particles contact point in the plane $x=0$, tangent to the particles spherical surfaces; $\chi$ is the potential barrier which the electrons cross and which equals the electron affinity of the particles surface (in vacuum).

We shall compare the thermal conductivities $k$ and $k_{V}$ of the structure and of a homogeneous semiconductor respectively. We shall assume that the electron tunneling happens mostly through the gaps $l<l_{c}<1 \mathrm{~nm}$ wide, that is through the area $s \approx \pi d_{c}^{2} / 4 \approx \pi l_{c} d / 2$, so $k / k_{V} \ll s / d^{2} \leq 10^{-4}$, where $d_{c}$ is the area diameter through which electrons mainly tunnel from one particle into another, and $l_{c}$ is the maximum gap between the particles that is tunnel-penetrable for electrons (Figure 1). If a constant temperature gradient $\nabla T$ along the particle chain is established, there emerge a temperature difference $\delta T$ and a potential difference $u$ between two particles. We can make an evaluation: $\xi / \xi_{V} \approx\left(k / k_{V}\right)^{1 / 2} \leq 10^{-2}$, where $\xi$ and $\xi_{V}$ are the temperature diffusivity coefficients of the structure and of a homogeneous semiconductor respectively. As $\xi / \xi_{V} \leq 10^{-2}$, temperature equalization rate within a particle is rather high, so when passing the border between particles in the contact area temperature changes stepwise by value $\delta T=T_{1}-T_{2} \cong d \nabla T$. Within each particle the solution of the stationary thermal conductivity equation $\Delta T=0$ shall be $T \cong$ const .

The electron fluxes through the contact region between two particles in the forward and backward directions coincide:

$$
i=i_{1} \exp \left\{-e u /\left(k_{B} T_{1}\right)\right\}-i_{2}=0 \text {, }
$$

where

$$
i_{1,2}=\left[n\left\langle v_{x}\right\rangle\right]_{1,2}=\iint_{0}^{d / 2} v_{x} f\left(\varepsilon, T_{1,2}\right) D[\varepsilon, l(r)] 2 \pi r \mathrm{~d} r \mathrm{~d}^{3} p,
$$

$e$ is the elementary charge; $k_{B}$ is the Boltzmann constant; $i_{1,2}=i_{1}$ or $i_{2} ; n$ is the electron concentration within a particle, $n=2\left(2 \pi m k_{B}\right)^{3 / 2}(2 \pi \hbar)^{-3} \exp \left[\left(F-E_{c}\right) /\left(k_{B} T\right)\right] ; F$ is the Fermi level; $E_{c}$ is the level of the conduction band bottom in the semiconductor; $v_{x}$ is the projection of the electron velocity vector onto the normal to the plane $x=0 ; \varepsilon=p^{2} / 2 m ; p$ and $m$ are the electron momentum and effective electron mass;

$T_{1,2}=T_{1}$ or $T_{2} ; f(\varepsilon, T)=n\left(2 \pi m k_{B} T\right)^{-3 / 2} \exp \left[-\varepsilon /\left(k_{B} T\right)\right]$ is the energy distribution function of electron. Using Expression (1), we shall find the differential thermopower of the material:

$$
\alpha=u /(d \nabla T)=\frac{k_{B} T}{e d \nabla T} \ln \left\{i_{1} / i_{2}\right\}
$$

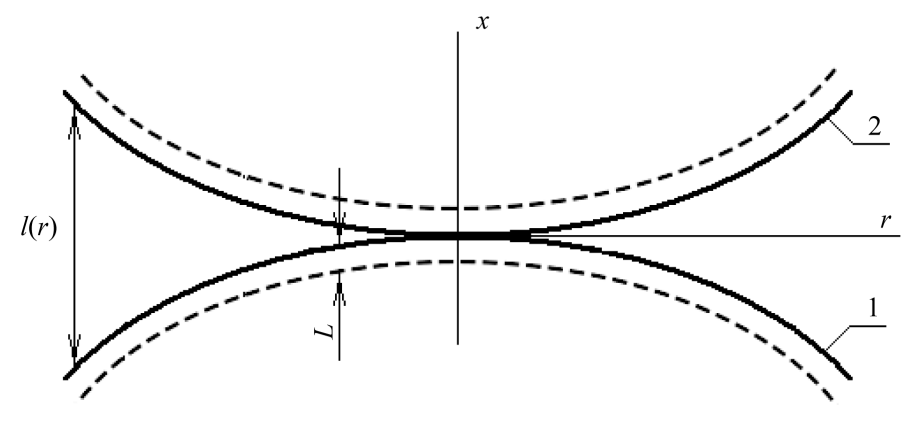

Figure 1. Schematic diagram of the contact between two identical ball-shaped particles 1 and 2: $l(r)$ is the gap between the particles at their contact, $L$ - the thickness of the semiconductor film. 
Let electric potential gradient $\nabla \phi$ be created in the material. We use the estimate $\sigma / \sigma_{V}<s / d^{2} \approx l_{c} / d<10^{-3}$ (see Figure 1), where $\sigma$ and $\sigma_{V}$ are the electrical conductivities of the structure and a homogeneous semiconductor, respectively. That means that the voltage drop within the particle is weak, the voltage drop in the contact area between two particles is $\Delta \phi \cong d \nabla \phi$. The electric current through the contact is (see Equation (1)):

$$
I=e i_{1} \exp \left\{-e(u-\Delta \phi) /\left(k_{B} T_{1}\right)\right\}-e i_{2} .
$$

If the condition $e \Delta \phi \ll k_{B} T$ is met, we use Equation (1) and the equality $4 I /\left(\pi d^{2}\right)=\sigma \nabla \phi$ and write the electrical conductivity of the material as

$$
\sigma \cong 4 e^{2} i_{1} /\left(\pi d k_{B} T\right) .
$$

The heat flow $q_{e}$, transferred by electrons through the contact zone of the two particles is calculated by the formula

$$
q_{e}=q_{1} \exp \left\{-e u /\left(k_{B} T_{1}\right)\right\}-q_{2},
$$

where

$$
q_{1,2}=\left[n\left\langle v_{x} \varepsilon\right\rangle\right]_{1,2}=\iint_{0}^{d / 2} v_{x} \varepsilon f\left(\varepsilon, T_{1,2}\right) D[\varepsilon, l(r)] 2 \pi r d r d^{3} p,
$$

$q_{1,2}=q_{1}$ or $q_{2}$. Using the expression $4 q_{e} /\left(\pi d^{2}\right)=k_{e} \nabla T$, we find the electronic thermal conductivity of the structure:

$$
k_{e}=4 q_{e} /\left(\pi d^{2} \nabla T\right) .
$$

The heat flow caused by the radiative heat exchange between two particles by means of thermal radiation and the radiant thermal conductivity of the material are then $q_{T}=S_{T} \sigma_{T}\left[(T+\delta T)^{4}-T^{4}\right]$, and

$$
k_{h}=q_{T} /\left(S_{T} \nabla T\right) \cong 4 \sigma_{T} T^{3} d,
$$

respectively with $\sigma_{T}$-the Stefen-Boltzmann constant.

Let us assume that the space between the particles is filled with a dielectric. In this case the potential barrier $\chi$ which the electrons cross equals the semiconductor and dielectric thermionic works difference. The heat flow $q_{p}$, transferred by phonons through the contact area between two particles can be found using the formula:

$$
q_{p} \cong 2 \pi k_{d} \delta T \int_{d_{c} / 2}^{d / 2} r l^{-1} \mathrm{~d} r=\pi k_{d} d \delta T \int_{d_{c} / 2}^{d / 2} r^{-1} \mathrm{~d} r \cong \frac{1}{2} \pi k_{d} d \delta T \ln \left(2 d / l_{c}\right),
$$

where $k_{d}$ is the dielectric thermal conductivity; $d_{c} \approx 2\left(l_{c} d\right)^{1 / 2} ; l_{c}$ is the gap between the particles were the dielectric molecules cannot enter. That means that the phonon thermal conductivity of the structure equals

$$
k_{p}=4 q_{p} /\left(\pi d^{2} \nabla T\right) \cong 2 k_{d} \ln \left(2 d / l_{c}\right) .
$$

Consider the thermoelectric properties of the material, consisting of a large number of identical spherical metal particles with a $n$-type semiconductor thin film on the surface thereof. We assume that the following conditions are met: $L \ll d ; \lambda \ll d$, where $L \leq 3 \mathrm{~nm}$-the thickness of the semiconductor film; $\lambda$-the de Broglie wavelength of electrons in the metal particles. In this case we have

$$
\begin{aligned}
& i_{1,2} \approx N_{c} \int_{\varepsilon<F+\Delta \varepsilon} v_{x m} f\left(\varepsilon, T_{1,2}, T_{2,1}\right) S_{m}(\varepsilon) \mathrm{d}^{3} p+N_{c} \int_{\varepsilon \geq F+\Delta \varepsilon} v_{x} f\left(\varepsilon, T_{1,2}, T_{2,1}\right) S(\varepsilon) \mathrm{d}^{3} p ; \\
& f\left(\varepsilon, T_{1,2}, T_{2,1}\right)=g\left(\varepsilon, T_{1,2}\right)\left[1-g\left(\varepsilon, T_{2,1}\right)\right] ; g(\varepsilon, T)=\left[1+\exp \left(\frac{\varepsilon-F}{k_{B} T}\right)\right]^{-1} ; \\
& S_{m}(\varepsilon)=\int_{0}^{d / 2} D_{m}\left[\varepsilon, E_{c}(x), l(r)\right] 2 \pi r d r ;
\end{aligned}
$$




$$
\begin{aligned}
& S(\varepsilon)=\int_{0}^{d / 2} D[\varepsilon, l(r)] 2 \pi r \mathrm{~d} r=\frac{\pi \hbar d}{4\left[2 m_{0}(\chi-\varepsilon)\right]^{1 / 2}}\left\{1-\exp \left[-\left(2 m_{0}\right)^{1 / 2} d(\chi-\varepsilon)^{1 / 2} / \hbar\right]\right\} \\
& D_{m}\left[\varepsilon, E_{c}(x), l(r)\right] \approx D_{\Phi}[\varepsilon, l(r)] \exp \left\{-\frac{4}{\hbar} \int_{x_{1}(\varepsilon)}^{x_{2}(\varepsilon)}\left\{2 m_{0}\left[E_{c}(x)-\varepsilon\right]\right\}^{1 / 2} \mathrm{~d} x\right\}, \varepsilon<F+\Delta \varepsilon \\
& D_{m}\left[\varepsilon, E_{c}(x), l(r)\right] \approx D_{\Phi}[\varepsilon, l(r)], \quad \varepsilon \geq F+\Delta \varepsilon ; \\
& D_{\Phi}[\varepsilon, l(r)] \approx \exp \left\{\frac{-2 l(r)\left[2 m_{0}(\Phi-\varepsilon)\right]^{1 / 2}}{\hbar}\right\}, \quad \varepsilon \leq \Phi ; D_{\Phi}[\varepsilon, l(r)] \approx 1, \varepsilon>\Phi ; \\
& q_{e} \approx q_{e S}+q_{e m}, q_{e s}=q_{1 S} \exp \left(\frac{-e u}{k_{B} T_{1}}\right)-q_{2 S} ; \quad q_{e m}=q_{1 m} \exp \left(\frac{-e u}{k_{B} T_{1}}\right)-q_{2 m} ; \\
& q_{1,2 S} \approx N_{c} \int \varepsilon v_{x} f\left(\varepsilon, T_{1,2}, T_{2,1}\right) S(\varepsilon) \mathrm{d}^{3} p, \quad \varepsilon \geq F+\Delta \varepsilon \\
& q_{1,2 m} \approx N_{c} \int \varepsilon v_{x m} f\left(\varepsilon, T_{1,2}, T_{2,1}\right) S_{m}(\varepsilon) \mathrm{d}^{3} p, \quad \varepsilon<F+\Delta \varepsilon \\
& I \approx e\left\{\begin{array}{c}
\left.i_{1} \exp \left[\frac{-e\left(u-\Delta \phi_{g}\right)}{k_{B} T}\right]-i_{2}\right\} \\
\sigma \approx \frac{4 e^{2} i_{1} P}{\pi d k_{B} T}
\end{array}\right.
\end{aligned}
$$

where $N_{c}=\left(4 \pi^{3} \hbar^{3}\right)^{-1} ; \Delta \varepsilon$-the potential barrier for electrons in the metal at the interface of the metal with the semiconductor film [10]; $v_{x m}$ and $v_{x}$-the projection of the velocity vector of the electrons in the metal and the semiconductor, respectively, to the direction normal to the plane $x=0 ; \quad x_{2}(\varepsilon)-x_{1}(\varepsilon) \leq L$-the width of the potential barrier; $\Phi$-thermionic work function of the surface of semiconductor films;

$\Delta \phi \approx \Delta \phi_{S}+\Delta \phi_{g} \approx d \nabla \phi ; \Delta \phi_{S}$ - the voltage drop across semiconductor films; $\Delta \phi_{g}$-voltage drop across the vacuum gap between the two particles; $P \approx\left(1+\Delta \phi_{S} / \Delta \phi_{g}\right)^{-1}$.

We now use the spherical coordinate system for the volume element $\mathrm{d}^{3} p=p^{2} \sin \theta \mathrm{d} \theta \mathrm{d} \phi \mathrm{d} p$ in the momentum space and the expression $v_{x}=p \cos \theta / m$. Integrating with respect to $\theta$ in the limits from 0 to $\pi / 2$ and with respect to $\varphi$ from 0 to $2 \pi$ and using Equation (10), (15) - (17), we find

$$
\begin{aligned}
& i_{1,2}=\frac{\pi N_{c}}{m}\left[\int_{0}^{p_{1}} p^{3} S_{m}(\varepsilon) f \mathrm{~d} p+\int_{p_{1}}^{p_{2}} p^{3} S(\varepsilon) f \mathrm{~d} p+\frac{\pi d^{2}}{4} \int_{p_{2}}^{\infty} p^{3} f \mathrm{~d} p\right], \\
& q_{1,2}=\frac{\pi N_{c}}{m}\left[\int_{0}^{p_{1}} \varepsilon p^{3} S_{m}(\varepsilon) f \mathrm{~d} p+\int_{p_{1}}^{p_{2}} \varepsilon p^{3} S(\varepsilon) f \mathrm{~d} p+\frac{\pi d^{2}}{4} \int_{p_{2}}^{\infty} \varepsilon p^{3} f \mathrm{~d} p\right],
\end{aligned}
$$

where $p_{1}=[2 m(F+\Delta \varepsilon)]^{1 / 2} ; \quad \Delta \varepsilon=E_{c}-F ; \quad f=f\left(\varepsilon, T_{1,2}, T_{2,1}\right) ; \quad p_{2}=(2 m \Phi)^{1 / 2} ; \quad \Phi=\chi+E_{c}-F$.

Expressions (1), (3), (7), (8), (9) and (11) - (20) were used to perform computer calculations of the values $\alpha$, $k, \sigma, k / \sigma$ and $Z=T \sigma \alpha^{2} / k$, with $k=k_{e}+k_{h}$ or $k=k_{e}+k_{h}+k_{p}$. Parameters $T ; \chi ; k_{d} ;\left(E_{c}-F\right)$ and $d$ were varied, values $\nabla T=10^{5} \mathrm{~V} / \mathrm{m} ; l_{c}=0.3 \mathrm{~nm} ; m=9.1 \times 10^{-31} \mathrm{~kg} ; P=1$ were constant.

Example of the calculated curves $Z(\chi), \alpha(\chi)$ at different film thicknesses $L$ nondegenerate semiconductor films on their surfaces are presented in Figure 2. By increasing $L$, the thermoelectric figure of merit of a material increases monotonically, approaching its limiting value.

We shall consider the thermoelectric properties of a material consisting of a large number of identical ballshaped non-degenerate $n$-type semiconductor particles contacting each other. In the range of values $\chi \geq 1.5 \mathrm{eV}$, 
the values $\alpha, k / \sigma$ and $Z$ are not dependent on $\chi$, if $T \approx 300 \mathrm{~K} ; k_{d}=0 ; 0 \leq\left(E_{c}-F\right) \leq 0.15 \mathrm{eV}$;

$d \approx 10^{-6} \mathrm{~m}$. Examples of the calculated curves $Z(d)$ and $Z(T)$ are presented in Figure 3 and Figure 4 . The maximum value $Z_{\max }=10^{2}$. If the particle diameter is enlarged while the other parameters are held constant at the $\chi \geq 1.5 \mathrm{eV}$, the value $Z(d)$ decreases monotonously (by a factor of ten or so) due to the increase of the radiant thermal exchange role in the material's thermal conductivity (see, for instance, Figure 3, curve 3 ). If the electron potential barrier $\chi$ is enlarged, the dependency $Z(\chi)$ includes the maximum at the $\chi^{*} \approx 0.4-0.7$ $\mathrm{eV}$, where $\chi^{*}$ depends on the temperature. Then, in the presence of an insulator between the particles $\left(k_{d} \geq 1\right.$ $\mathrm{W} / \mathrm{m} \cdot \mathrm{K}), Z(\chi)$ continues to decrease rapidly and monotonically.

In the range $\chi \geq \chi^{*}$ these calculated $\chi$ independent values are equal to the values calculated by the formulas:

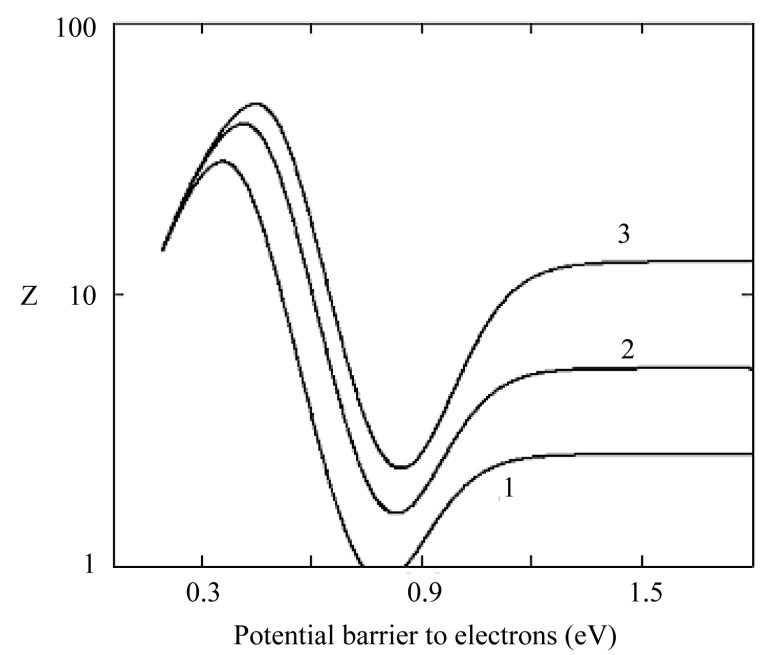

Figure 2. Thermoelectric figure of merit of a material consisting of metal particles vs. the potential barrier to electrons tunneling through the gap between the particles with different thicknesses of the semiconductor films on the surface of the metal particles. $L=(1) 0$, (2) 1 and (3) $3 \mathrm{~nm} . D=10^{-6} \mathrm{~m} ; T=700 \mathrm{~K} ; E_{c}-F=0.2 \mathrm{eV} ; k_{d}=0$.

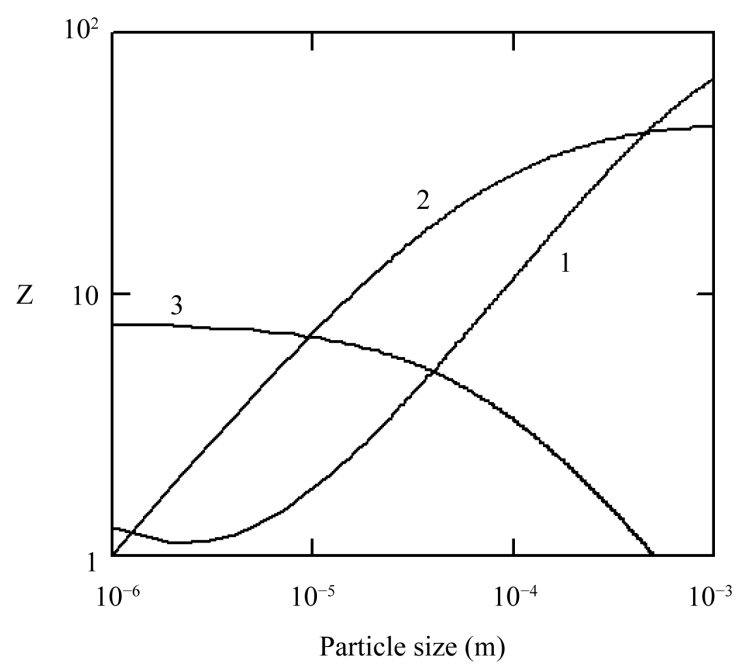

Figure 3. Thermoelectric figure of merit of a material consisting of semiconductor particles vs. the particles diameter with other parameters held constant. $\left(E_{c}-F\right)=(1)$, (2) and (3) $0.05 \mathrm{eV} ; \chi=(1) 0.7$, (2) 0.3 and (3) $1.5 \mathrm{eV} ; T=(1)$, (2) 600 and (3) $300 \mathrm{~K}$; $k_{d}=(1)$, (3) 0 and (2) $1 \mathrm{~W} /(\mathrm{m} \cdot \mathrm{K})$. 


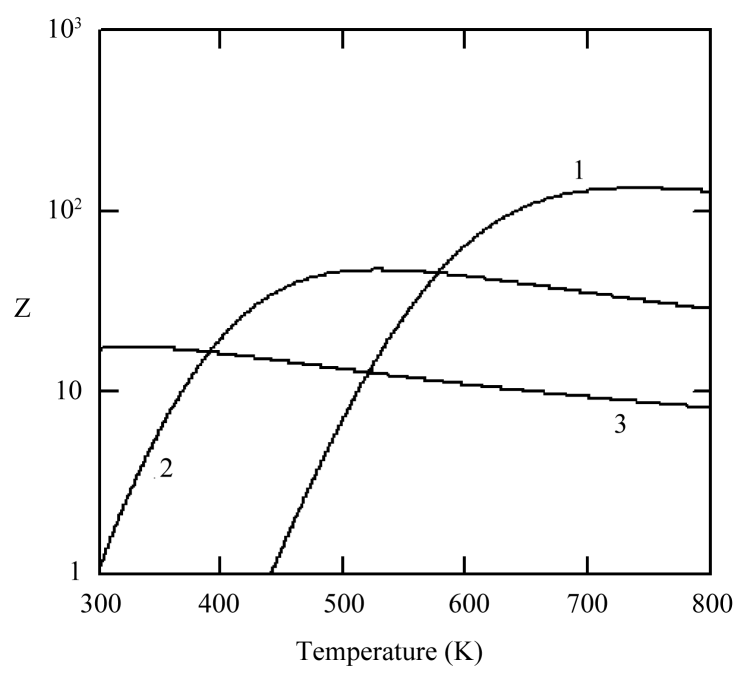

Figure 4. Thermoelectric figure of merit of a material consisting of semiconductor particles vs. the temperature of a material. $\left(E_{c}-F\right)=$ (1), (3) 0.15 , and (2) $0.05 \mathrm{eV} ; \chi=$ (1) 0.7 , (2) 0.3 and (3) $2 \mathrm{eV} ; d=$ (1), (2) $10^{-3}$ and (3) $10^{-6} \mathrm{~m} ; k_{d}=(1)$, (3) 0 and (2) $1 \mathrm{~W} /(\mathrm{mK})$.

$$
\alpha=2 k_{B} / e+\left(E_{c}-F\right) /(e T), k / \sigma=2 k_{B}^{2} T / e^{2}, Z=\left[2+\left(E_{c}-F\right) /\left(k_{B} T\right)\right]^{2} / 2
$$

(see, for instance, Figure 3 and Figure 4, curves 3). We can demonstrate that those formulas can be found with the use of the Formulas (1) - (7) if the following conditions are met $k=k_{e}$ and in the Formulas (2), (6) $D[\varepsilon, l(r)]=\bar{D}=$ const .

\section{Conclusion}

Thus the currently existing semiconductor particles can be used to produce structures with the thermoelectric figure of merit $Z=10$ - 100, if the thermionic work function of the particle surface $\Phi$ is lower than $1 \mathrm{eV}$. If the semiconductor particles diameter $\left(d \geq 10^{-5} \mathrm{~m}\right)$ is enlarged and $\Phi \leq 1 \mathrm{eV}$ while the other parameters are held constant, the value $Z(d)$ increases monotonously. When the temperature of a material increases, the thermoelectric figure of merit increases, if $\Phi \leq 1 \mathrm{eV}$. By increasing thicknesses of the semiconductor films on the surface of the metal particles, the thermoelectric figure of merit of a material increases monotonically, approaching its limiting value.

\section{Acknowledgements}

This work was supported by the Russian Foundation for Basic Research (project no. 12-02-97500) and the authorities of Orel oblast.

\section{References}

[1] Dmitriev, A.V. and Zvyagin, I.P. (2010) Current Trends in the Physics of Thermoelectric Materials. Physics Uspekhi, 53, 789-803. http://dx.doi.org/10.3367/UFNe.0180.201008b.0821

[2] Snarskii, A.A., Sarychev, A.K., Bersudnov, I.V. and Lagarkov, A.N. (2012) Thermoelectric Figure of Merit of Bulk Nanostructured Composites with Distributed Parameters. Semiconductors, 46, 659-665. http://dx.doi.org/10.1134/S106378261205020X

[3] Casian, A.I. (2006) In: Rowe, D.M., Ed., Thermoelectrics Handbook: Macro to Nano, CRC/Taylor and Francis, Boca Raton, Chapter 36.

[4] Kharlamov, V.F. (2013) Thermoelectric Figure of Merit of a Material Consisting of Semiconductor or Metal Particles. Journal of Experimental and Theoretical Physics, 117, 83-88. http://dx.doi.org/10.1134/S106377611306006X

[5] Gothard, N., Spowart, J.E. and Tritt, T.M. (2010). Physica Status Solidi (A), 207, 157-164.

[6] Ohita, H. (2007) Thermoelectrics Based on Strontium Titanate. Materials Today, 10, 44-49. 
http://dx.doi.org/10.1016/S1369-7021(07)70244-4

[7] Tritt, T.M. and Subramanian, M.A. (2006) Thermoelectric Materials, Phenomena, and Applications: A Bird's Eye View. MRS Bulletin, 31, 188-197. http://dx.doi.org/10.1557/mrs2006.44

[8] Zhu, G.H., Lee, H., Lan, Y.C., Wang, X.W., Joshi, G., Wang, D.Z., Yang, J., Vashaee, D., Guilbert, H., Pillitteri, A., Dresselhaus, M.S., Chen, G. and Ren, Z.F. (2009) Increased Phonon Scattering by Nanograins and Point Defects in Nanostructured Silicon with a Low Concentration of Germanium. Physical Review Letters, 102, 196803. http://dx.doi.org/10.1103/PhysRevLett.102.196803

[9] Hishinuma Y., Moyzhes B.Y., et al. (2001) Refrigeration by Combined Tunneling and Thermionic Emission in Vacuum: Use of Nanometer Scale Design. Applied Physics Letters, 78, 2572. http://dx.doi.org/10.1063/1.1365944

[10] Bonch-Bruevich, V.L. and Kalashnikov, S.G. (1982) Physics of Semiconductors. VEB, Berlin. 
Scientific Research Publishing (SCIRP) is one of the largest Open Access journal publishers. It is currently publishing more than 200 open access, online, peer-reviewed journals covering a wide range of academic disciplines. SCIRP serves the worldwide academic communities and contributes to the progress and application of science with its publication.

Other selected journals from SCIRP are listed as below. Submit your manuscript to us via either submit@scirp.org or Online Submission Portal.
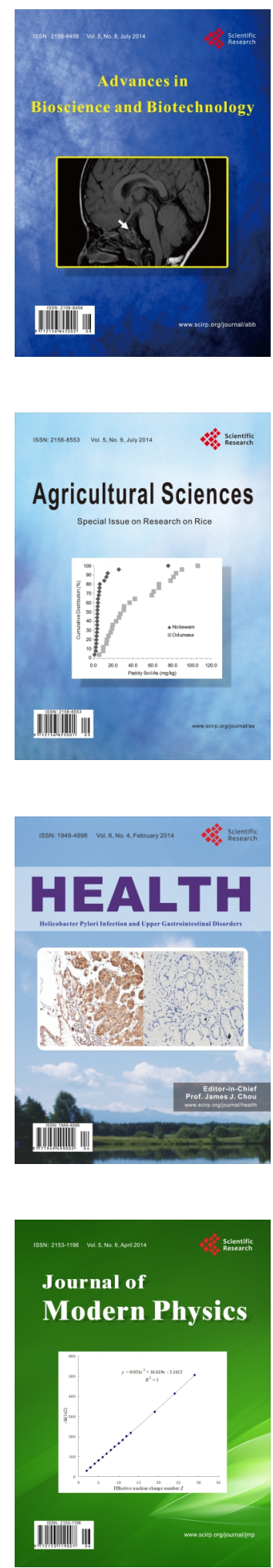
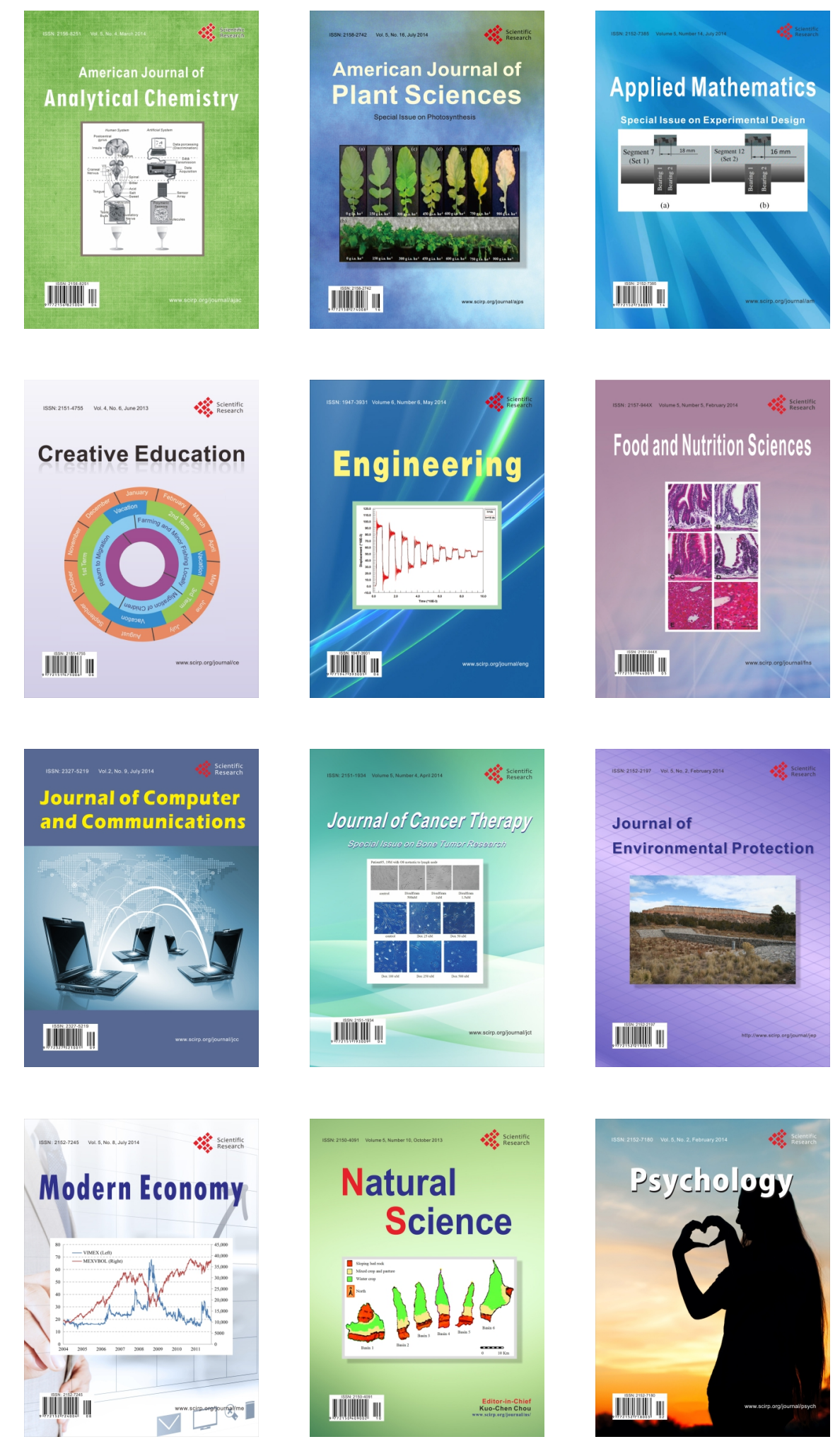